\title{
Usos del término ministra: De la sociedad romana a las comunidades cristianas
}

\author{
Ma Daría SaAvedra-Guerrero *
}

La razón de este artículo se encuentra en nuestro interés por conocer la evolución en el uso de determinados vocablos y como mantienen a lo largo del tiempo sus valores semánticos. Para este propósito hemos elegido el término ministra porque en él se dan determinadas características como son el mantener sustancialmente su significado a lo largo del tiempo, utilizarse para designar diferentes cargos y oficios y contar con el valor añadido de ser el origen de una peculiar división de opiniones entre historiadores, filólogos y canonistas.

Nos encontramos ante una denominación que existe desde antiguo en Roma ${ }^{1}$, y que sirve para identificar a aquellas mujeres, esclavas y libertas, que ejercen el oficio de servir la mesa, a aquellas que desempeñan una magistratura dentro de las asociaciones y se encargan de ayudar a sus presidentes - magistri- y a las que se encuentran dentro de la jerarquía sacerdotal en los templos dedicados al culto de diversos teónimos paganos. Con el tiempo se trasladará a un contexto religioso-cultural de origen extrarromano y se utilizará para identificar un oficio desempeñado por algunas mujeres en las incipientes comunidades cristianas.

\section{LA SOCIEDAD ROMANA: LA VILLA, EL COLLEGIUM, EL TEMPLUM.}

Entre las múltiples actividades que en la sociedad romana podía realizar una mujer trabajadora ${ }^{2}$ encontramos a algunas ministrae dentro de las

\footnotetext{
Universidad de Cantabria.

Vid. Christine MOHRMAN: «Latin langue de la chretienté occidentele» en ID: Efudes sur le latin des chretiennes. Roma, 1961. vol 1. pp. 62-63.

2 Vid. J. LE GALL: «Metiers de femmes au Corpus Insciptionum Latinarum». REL 47, bis. (Melanges Marcel Durry), 1960. pp. 123-130. Susan TREGGIARI: "Jobs for women» AJAH1, 1976. pp. 76-104. Natalie KAMPEN: Image and status: Roman working women in Ostia. Berlin, 1981.
} 
casas, cuyo equipo doméstico reflejaba una considerable división del trabajo, con los oficios más diversos ${ }^{3}$ en manos de esclavos, necesarios para el buen funcionamiento del hogar de los patroni. Sus responsabilidades se centraban en el servicio de la mesa ${ }^{4}$ o en el cuidado del vestuario ${ }^{5}$. En las fuentes epigráficas aparecen identificadas con la expresión ministra in familia ${ }^{6}$ para diferenciarlas de las ministrae collegiorum y de las ministrae sacrorum $^{7}$.

Los collegia toman su estructura interna y sus magistraturas del municipium en sus aspectos administrativos, económicos y religiosos ${ }^{8}$. En cambio, es dificil saber de donde toman los collegia el título de ministra, puede ser que del mundo doméstico, una hipótesis factible puesto que en las asociaciones existen miembros en sus listas de la más diversa condición jurídica. Pueden haber tomado su modelo de la jerarquía religiosa, cuestión verosímil debido a la importancia que tiene el factor religioso ${ }^{9}$ en las actividades de una asociación sin que haya de ser específicamente religiosa ${ }^{10}$ y a los contínuos contactos que tenían algunos collegia con los templos de

3 Susan Tregglarl: "Questions on Women domestics in the Roman West» en Schiavitú, manomisione e classi dipendenti nel Mondo antico: Universitá degli studi di Padova. Pubblicazioni dell'Instituto di Storia antica XIII. Roma, 1979, pp. 185-201. ID: «Jobs for women»... J. LE GALL: op. cit. Natalie KAMPEN: op. cit.

4 Esclavas: CIL VI, 9290 b, 9637, 9638a, 9639. Liberta: VI, 9640. Cfr. Natalie KamPEN: op. cit., p. 120.

5 Vestispica: VI, 9901 (esclava imperial). Cfr. Natalie KamPEN: op. cit., p. 120. Vestisplicae: VI, 37202 (esclava), 9912 (posiblemenle esclava), 37302b (liberta). Cfr. Natalie KampEN: op. cit, p. 120.

6 VI, 9637: Nice minor / ministra in familia. VI, 963X a.: Vipsania Soteris / ministr(a) in familia / lalysi et Asmeti / mater.

$7 \mathrm{X}, 4791$.

\& Para el conocimiento general del fenómeno asociativo vid. J. P. WAL.TZING: Étude historique sur les corporations professionelles chez les romains. Lovaina, 1898-1900-,Roma, 1968. 4 vol. F. M. DE RoBERTIS: // dirito associtivo romano, dai collegi della Republica alle corporazioni del Basso Impero. Bari, 1938. ID.: II fenomeno associativo nel mondo romano. Dai collegi della republica alle corporazioni del Basso Impero. Roma, 1981. ID.: Storia sociale di Roma. Le classi inferiori. Contributi varii alla storia economica e social di Roma. Roma, 1981. ID.: Lavoro e lavoratori nel mondo romano. Bari, 1963. ID.: Storia delle corporazioni e del regime associativo nel mondo romano. Bari, 1971. L. HARMAND: Le patronat sur les collectivités publiques des origines au BasEmpire. Paris, 1957. Lellia CRACCO-Ruggini: "Le assoziacioni nell mondo romano bizantino". Atti della XVIII settimana di studi sull'Atto Medioevo. Spoleto, 1971. pp. 59 193. ID.: «La vita associativa nelle città dell'Oriente greco: tradizioni locali e influenze romane". Assimilation et resistánce á la culture greco-romaine dans le monde ancien. Travaux du Vle Congrès International d' Etudes classiques. Madrid, 1974; Paris, 1976. pp. 463-491.

9 Esta relación llega a ser tan importante en ocasiones que algunos colegios utilizan para la realización de sus cultos religiosos templos públicos. Cfr. J. P. WALTZING: op. cit, vol. IV (Indices); pp. 454-455.

10 Para conocer los diversos tipos de asociaciones vid. Vid. J. P. WALTZING: op. cit., 1968. I pp. 42-48. F. M. DE ROBERTIS: II dirito associativo romano, dai collegi della Republica alle corporazioni del Basso Impero. Bari, 1938, p. 6. 
su ciudad, hasta tal punto de llegar a cederles el edificio para sus ritos religiosos ${ }^{11}$. Lo que sí sabemos es que mantiene su significado ya que las ministrae collegiorum ${ }^{12}$ son las ayudantes del presidente, el magister. No es muy frecuente encontrarnos ministrae en las titulaturas colegiales como tampoco lo es la presencia femenina en el fenómeno asociativo en general ${ }^{13}$, pero no por ello hay que restarle importancia. En general se puede considerar que la presencia de ministri en una asociación es un indicio de la existencia de miembros de condición jurídica distinta, esclavos ${ }^{14}$ y libertos ${ }^{15}, \sin$ que por ello se deba deducir que fueran los esclavos propiedad del colegio ${ }^{16}$.

Esta magistratura aparece en asociaciones de tipo asistencial como son las que tienen, entre sus fines principales, el proporcionar un lugar de enterramiento a sus asociados. Se les conoce como collegia tenuiorum y en uno de ellos, radicado en Roma, nos encontramos ${ }^{17}$ a la ministra Ossua Atalene inscrita tras sus colegas magistri y tribuni. En la misma ciudad y procedente de un monumentum del s. I d. C. tenemos un texto, escrito en sus dos caras, en el que nos encontramos, en la cara A a Vivo Quartio textor, (trium)vir, questor, trib(unus) y en la cara B a Hilara Minor, ministra ${ }^{18}$. Esta establece que sus cenizas sean colocadas en la misma olla que Midas, cubicularius (trium)vir. Ella es quien hace la dedicatoria en la que insiste en su deseo de ser enterrada junto a su collega: «ut ossa sua in olla Midaes coicirentur cum mort(ua) esset ${ }^{19}$. Este factor personal excede

11 Cfr. J.P. WALTZING: op. cit., vol. IV (Indices); pp. 437-442.

12 Las funciones de los ministri no están determinadas específicamente quedando encerradas en el concepto ambíguo y genérico de ayudante, debiendo entender que la asignación de sus responsabilidades estaban en manos del colegio del que se tratase. Vid. J.P. WALTZING: op. cit., vol. I. pp. $422-423$.

13 Vid. Ma Daría SaAvedra-Guerrero: La mujer $y$ las asociaciones en el imperio romano. Servicio de publicaciones de la Universidad de Cantabria. Santander, 1991. (Tesis doctoral en microficha). passim. J.P. WALTZING: op. cit, vol. VI (Indices); pp. 254257.

14 Administran juntos el colegio, la diferencia estriba en la condición jurídica de quien desempeña la función. Ministri: I, $1129=$ XIV, 2874; XIV, 2982; X, 6679; VI, 10312; VI, 10330; XI, 5737; I, p. 159; X. 3789; X, 824-828, 885, 910, 924, 137, 205, 1269, 4789, 4791, 7953. Cfr. J.P. WALTZING: op. cit., l; pp. 422-423.

15 Cuando hay libertos y esclavos, los primeros son los magistri y los segundos ministri aunque no siempre, puesto que existen colegios donde hay esclavos que son magistri (XIV, 2875). Cfr. J.P. WALTZING: op. cit., IV (Indices); p. 251.

16 Existen datos de la coexistencia de esclavos y libertos en un mismo colegio sin que por ello deba entenderse que los esclavos lo eran de la asociación sino que se trata de miembros de número al igual que el resto de los colegiales. Vid. J.P. WALTZING: op. cit., IV (Indices); pp. $251-254$.

17 VI. 10311.

18 I, 9290 (Tabla de mármol, escrita con pequeños caracteres, procedente de lo que debió ser un columbario). Hasta la V1, 9294 pertenencen a un mismo monumentum fechado en el 9 . d. C. con presencia mayoritaria de esclavos.

${ }_{19}$ VI, 9290 b, 8. 
lo que debieron ser sus funciones ministeriales, el encargarse de la adjudicación de un locus en el columbarium colegial y, probablemente, de que se rindieran los ritos pertinentes al difunto.

En su faceta religiosa las ministrae aparecen inmersas en la jerarquía sacerdotal dedicada al culto de algunos teónimos romanos, preferentemente de fidelidad femenina ${ }^{20}$, como las que están dedicadas al culto de la Bona Dea, Rufia Festa, Caesilia Festa, Caesilia Scylace, Decidia Paulina y Pupia Peregrina ${ }^{21}$, Caiena Attica ${ }^{22}$ y la ministra de la Magna Mater, Acca Prima ${ }^{23}$. Tambien aparecen en el culto imperial como la hispana Vibia Felicula ${ }^{24}$ ministra del culto de la Paz Perpetua y de la Concordia Augusta y así como en otros epígrafes en lo que no hay evidencia de estar vinculadas a un culto concreto ${ }^{25}$. La actividad religiosa de las ministrae quedan reflejadas en algunos textos literarios ${ }^{26}$ que ponen de manifiesto su uso en un contexto pagano en el cual a las mujeres se les asigna una función sin determinar en el rito, pero en la que con toda probabilidad se mantiene la idea de ayuda y asistencia en el desarrollo del culto.

\section{LAS COMUNIDADES CRISTIANAS}

Tras la muerte de Jesús sus discípulos directos y sus seguidores se ponen en marcha con el fin de difundir su mensaje ${ }^{27}$ por todo el mundo conocido. El progresivo éxito de su proyecto hizo que surgieran en las diferentes ciudades visitadas por los apóstoles infinidad de comunidades al abrigo de los domicilios de algunos de sus primeros adeptos. En este contexto se experimentará la conversión de una secta del judaismo en una religión con personalidad propia. Esto lleva implícita la formación de una estructura interna independiente que toman prestada de su entorno

20 Vid. J. Gage: Matronalia. Essai sur les devotions et les organisations cultuelles des femmes dans lancienne Rome. Bruselas, 1963.

$$
\mathrm{V}, 762 .
$$

XII, 654.

23 IX, 3146.

24 II, 3349: Augusto / Paci Perpetuae et Concordiae / Augustae / Q(uintus) Vibius Setino sevir et / Vibia Felicula ministra Tutelae / Augustae / d. s. p. d. d. Sobre el culto imperial en Hispania Vid. R. ETIEnNE: Le culte imperial dans le Peninsule lbérique d'Auguste à Diocletien. Paris, 1974.

$25 \mathrm{~V}, 5026$ : listado de magistrae y ministrae. $X, 4791$ : ministrae sacrorum.

26 «una ministratum» OV.: Met., 9, 90; 306; 14, 705. «ara dea certe tremuit, pariente ministra» (se refiera a su pariente la vestal Silvia). ID. Fast, 3, 47; 6, 283, 289, 441. "accipiat missas apta ministra notas» ID. Ars. Am., 3, 470. CATUL., 63, 68. SERV., Aen., 2, 735. PROP. 4, 4, 44. Cfr. Thesaurus linguae latinae. Leipzig, 1936-1966. sv. «ministra».

${ }_{27}$ Hech., 4, 4; 8, 4; 15, 7; 16,14;17, 4-12; 28, 28. 
más próximo asignando términos y responsabilidades que les son afines. Así es facil pensar que algunas de las funciones tomaran su denominación del entorno doméstico en el que germinaron las primeras comunidades, tambien llamadas ecclesiae ${ }^{28}$, un ambiente en el que, como en cualquier casa que se preciara de tener cierto nivel económico ${ }^{29}$, contaba con relativo número de esclavos encargados de su funcionamiento diario y de la asistencia a sus habitantes. Estas iglesias son mencionadas frecuentemente por San Pablo en sus cartas ${ }^{30}$ y en las que además se esboza la participación de mujeres ${ }^{31}$ codo con codo con los difusores del cristianismo ${ }^{32}$. A la hora de asignar nombres a las distintas funciones es lógico que se acudiera a lo que se encontraba más próximo al entorno en que discurria habitualmente la vida de esas comunidades, adscritas a la nueva religión que, si bien no eran secretas, sí se mantenían discretas y alejadas de la curiosidad general, en el ambiente privado de una casa familiar ${ }^{33}$.

En este escenario doméstico de judíos helenizados y ricos, es fácil que existiera entre sus esclavas alguna que desempeñara una función equivalente a la desarrollada por las ministrae en el occidente latino y que fuera adoptada como denominación en la incipiente jerarquización

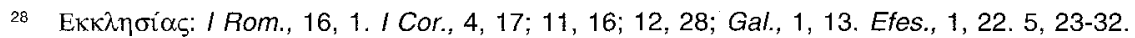
Filip., 3,6 .

29 «Pomponia Graecina, insignis femina, Plautio, qui ovans sede Britannis rettulit, nupta ac superstitionis externae, mariti judiciio permissa. Isque prisco instituto propinquis aram de capito famaque coniugis cognovit, in insontem nuntiavit». TAC. Ann., XIII, 32, 2-3. Cfr. B. AuBE: Les chretiens dans l'empire romain. Roma, 1972; p. 248.

30 A lo largo de las cartas de S. Pablo, especialmente en sus apartados finales dedicados a dar mensajes de tipo personal, nos encontramos con detalles relacionados con el cobijo que algunos cristianos - se mencionan a los dos miembros de la pareja- daban en sus casas para las reuniones de la comunidad de su ciudad. Vid. I Rom. 16, 3-s.; / Cor. 16, 19. Col. 4, 15.

31 Esto se mantiene en las cartas auténticas porque en las deuteropaulinas, cuando se habla del papel que deben desempeñar las mujeres en las comunidades cristianas, se frena y se da marcha atras y, de reflejar una situación de actividad conjunta, se empieza a dar normas que relegan a las mujeres a un papel tradicional, secundario y silencioso entre los cristianos (l Tim., 2, 812) que nos recuerda la conducta seguida por las mujeres en la cultura judía. Vid. L.J. ARCHER: «The role of the Jewish women in the religion, ritual and cult of Graeco-Roman Palestine» en A. Cameron- A. Kurth (ed.): Images of Women in Antiquity. Londres, 1984. p. 274. A. Momigliano: "Macrina: una santa aristocrática vista dall'fratello. Appendice: "ll posto della donna nelle synagoge greche ellenistiche» en G. ARRIGoN! (ed.): Le donne in Grecia. Roma-Bari, 1985. p. 340. Bernardette J. Brooten: Women Leaders in Ancient Synagoge. Inscriptional evidence and background issues. Chico, California Scholars Press, 1982.

32 Hechos. 5,$14 ; 8,3 ; 9,2 ; 17,4 ; 12,34$.

33 Se trata de judios helenizados en la diáspora. En relación con la existencia de comunidades cristianas con sede en domicilios particulares y su conexión con antecedentes judios vid. J. MontSerRAT TORRENTS: La sinagoga cristiana. El gran conflicto religioso del s. I. Barcelona, 1989. pp. 108-110. 
de los grupos de cristianos en su equivalente en la lengua en están escritos los primeros textos del periodo apostólico. Hasta bien avanzado en s. Il d. C. no se usará el latín como lengua literaria para las cristianos ${ }^{34}$.

Por esta razón nos vamos a encontrar para este oficio de servicio dos términos distintos, en griego y en latín, identificadores de una misma función profesional. Así, para los textos de los primeros tiempos del cristianismo, aparecen indistintamente, el sustantivo $\delta \alpha^{\alpha}$ kovo, , $0, \eta^{35}$ y el verbo $\delta$ ¿ckơvé $\omega$ aunque sea este último el más frecuente, especialmente en los Evangelios ${ }^{36}$ con el que se expresa la actividad del servidor, sobre todo en la mesa ${ }^{37} \mathrm{y}$, en un sentido más amplio, sirve para indicar disponibilidad, incluso de caracter espiritual.

San Pablo, en la primera carta a los Romanos ${ }^{38}$, en el momento de las despedidas, recomienda a una mujer, de nombre Febe, miembro de la iglesia de Cencres y que parece que va a desplazarse a la capital, con el fin de que sea bien atendida por los cristianos de Roma ${ }^{39}$. Ella parece ser una benefactora ${ }^{40}$ de la comunidad de Cencres, ciudad próxima a

34 Tertuliano (c. 160-225 d.C. ). Vid. J. FONTAINE: La letteratura latina cristiana. Bolonia, 1973. pp. 29-ss. A. Hamman: Dictionnaire des peres de l'Eglise. Paris, 1977. s. v. "Tertullien".

35 Su traducción en latín: minister, ministrator, famulus, ministra, ministratrix, famula, Cfr.

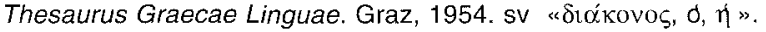

36 In., 12, 16. Cfr. A. G. Montinmont: Les diaconisses. Essai historique. Roma, 1982. p. 15.

37 Esto nos pone en relación con la actividad desempeñada por las esclavas domésticas encargadas de servir la mesa conocidas como ministrae y de aquellas vinculadas al culto religioso, preferentemente de caracter femenino.

38 Vid. J. D. G. DunN: "Paul's epistle to the Romans: an analysis of structure and argument" ANRW II 25.4, 1987. pp. 2842-2890. (cuenta con una amplia bibliografia). M. J. LAGRANGE: Saint Paul, Epitre aux Romains. Paris, 1950. p. 360.

39 Se trata de una carta de recomendación. Vid. J. D. G. DunN: op. cit., p. 2.883. Sobre este apartado de la carta a los Romanos hay quien llega a cuestionarse si se trata de un fragmento independiente J. I. H. MCDONNALD: «Was Romans 16 a separate letter? New Testament Studies 16.1969-1970. pp. 369-372.

40 El término que se le aplica es $\pi \rho \circ \sigma \tau \alpha \dot{\tau}$, , protectora con un sentido legal. Se aplica a aquella persona que ejerce la representación de extranjeros privados de garantías jurídicas. Cfr. M. J. LAGRANGE: $o p$. cit, p. 362. De otra manera la protección, aunque probablemente sin ese matiz, no constituye un fenómeno aislado puesto que a lo largo de las cartas de $\mathrm{S}$. Pablo nos encontramos con expresiones de agradecimiento a diversos benefactores de las comunidades a las que dirigía sus mensajes, especialmente aquellos que prestaron sus casas para sus reuniones y a mujeres que trabajaban en bien de la comunidad sin que sepamos concretamente en qué, dado que el término

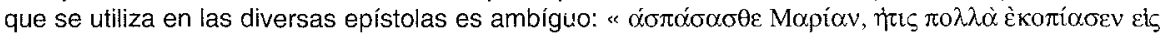

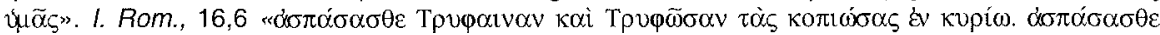

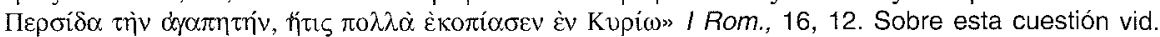
WAYNE A. MEEKS: The Firts Urban Christians. the Social World of the Apostle Paul. New Haven, 1983 pp. 60-61. MARIE ROSE D'ANGELO: «Women in the Luke-Acts. A relational View». JLB 109, 1990, pp. 441-461. Ross SHEPARD KRAEMER: Her Share of the Blessings. Women's Religions among Pagans, Jews and Cristians in the Greco-Roman World. Nueva York, 1992, p. 174. 
Corinto, y con la que el apóstol ha tenido contacto directo pues habla de la ayuda que ha recibido personalmente de ella. El texto original dice: « $\Sigma v v$

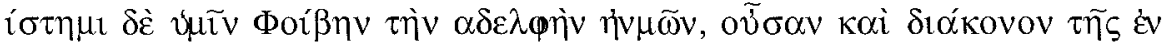

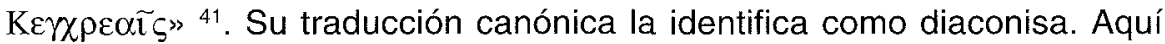

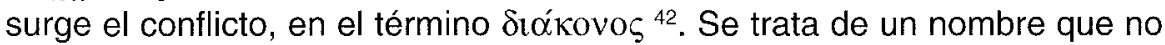
tiene desinencia femenina y que significa servidor, ministro ${ }^{43}$, siendo el único caso en el que se asocia con una mujer el ejercicio de esta función. La duda se plantea cuando hay que trasladarlo al latín. Hay quienes optan

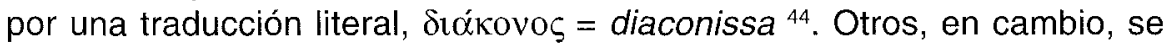
dirigen hacia una formulación genérica que la aproximaría más hacia el término latino ministra, esto es, $\delta$ cókovov = quae est in ministerio ${ }^{45}$. $\mathrm{Y}$ otros no consideran conveniente la traducción literal del término griego y estarían más cercanos a la que encontramos en el Thesaurus linguae graecae de $\delta$ iókovo $\varsigma=$ ministra ${ }^{46}$.

En los inicios del s. II d. C. (111 d. C.) Plinio el Joven, siendo gobernador de Bitinia, habla de dos ministrae esclavas a las que tortura con el fin de saber la verdad de las sectas cristianas: «Quo magis necessarium credidi ex duabus ancillis, quae ministrae dicebantur quid esset veri et per tormenta quaerere" ${ }^{47}$. Esta referencia es importante por la mención que se hace de dos términos coincidentes en las dos mujeres y variables

41 IRom. 16,1.

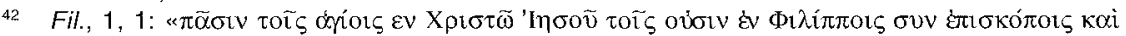

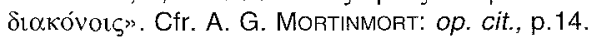

43 Roger GRYson: op. cit.,. p. 22. Los cristianos latinos, del s. IV d. C. y posteriores, traducí-

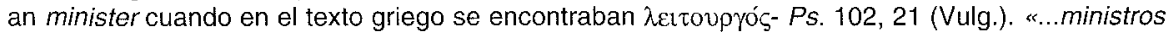
quos vocamus angeles». LACT. Div. Inst. 1, 7, 4; 1, 7, 8. ID. De mort pers., 4, 9, 5. EPIT., $22,3$. En otros casos nos encontramos con que ministerium se utiliza como sinónimo de minister. TERTUL. Apol., 39, 2. Rufin. Hist. mon., 29. Cfr. Cristine Mohriman: «Exultan divina ministeria» en ID: op. cit., vol I. pp.223-231.

44 A. LECLERCQ defiende la idea canónica de diaconado para el caso de Febe. CarRolLLECLERCQ: DACL. Paris, 1922. s.v. diaconesses, p. 725. J. Forget defiende la idea de la existencia del diaconado desde la época apostólica. ID.: “Diaconesses» DTC 4, pp. 685-703. Cfr. Roger GrYson: op. cit., p. 22. J. G. DAvIES: «Deacons, deaconesses and minor orders in the patristic period", Journal of ecclesiastical history 14,1963.

45 Traducción en sentido amplio que adopta la Vulgata. Cfr. M. J. Lagrange: op. cit., p. 362. Otros exégetas siguen la misma línea y lo traducen como que sirve en la iglesia de Cencres. Traduction oecumenique de la Bible, Noveau Testament. Paris, 1973. p. 487. Cfr. A. G. Montinmort: op. cit., p. 15.

46 Para aquellos que entienden que no debe traducirse el término júkovos por diaconisa sino como el término latino ministra, Vid. Christine MoHRMAN: «Latin langue de la chretienté...., 1. pp. 51-81. A.G. Mortinmort: op. cit, pp. 14-16. Roger Gryson: Le ministère des femmes dans l'eglise chretienne. Paris, 1971. pp. 38-39.

47 PLIN. Ep., 10, 96, 8 (ad Traianum). A. N. SHERWIN-WHITE: The letters of Pliny: historical an social commentary. Oxford, 1966. 
según quien sea el que las denomine. Para él son ancillae, que para un romano es indicativo de su condición social, dado que este término tiene un marcado caracter jurídico. Para sus correligionartos son además ministrae ${ }^{48}$. Desgraciadamente Plinio no muestra interés en expresar qué función específica desempeñan, por otro lado, secundario para sus intereses, quien está describiendo una situación a la que ha tenido que enfrentarse como gobernador y de la que está dando cuenta al emperador Trajano. Desde el punto de vista filológico y semántico este fragmento tiene un valor especial ya que tenemos la equivalencia de dos términos usados en dos medios socioculturales distintos, en primer lugar Plinio hace referencia con el término ancilla a su condición social ${ }^{49}$ de esclavas y en segundo lugar expresa cómo se denomina su función entre los cristianos.

La idea de servicio y ayuda ${ }^{50}$ se mantiene para el término ministra más allá de la época apostólica. Aparece en algunos autores cristianos que le utilizan con ese sentido como hace en sus cartas Paulino de Nola ${ }^{51}$.

Como hemos visto en el uso del término ministra nos encontramos con un hilo conductor que traslada la idea de ayuda, servicio y auxilio a lo largo del tiempo, de un contexto a otro, de una cultura a otra y de una religión a otra. Todos los factores, la villa, el collegium y el templum hacen que, ya sea en un medio plenamente romano o en un medio judío helenizado, podamos considerar que semánticamente estamos ante dos denominaciones, ministra y su traducción griega $\delta$ í́kovos, que podemos consiciarar equivalentes al menos en los primeros tiempos del cristianismo y, eso sí, antes de que al término griego se le asigne una función específica dentro de la jerarquía en las comunidades cristianas reservado a las viudas ${ }^{52}$.

48 Hay quien considera que Plinio hace del término diaconissa una trasposición mecánica a ministra. Cfr. Francesco Trisoglo (ed.) PLINIO: Epistolarum. Turín, 1973. vol. II, p. 1.097, n. 632.

49 R. Gryson: op. cit., p. 39. Para los cristianos se trata de un título dado a quienes han hecho profesión de vida religiosa. Cfr. Criarlton T. LEwIs: A latin dictionnary. Oxford, 1980. Vid. Carroll-LeClerq: DACL. Paris, 1922. s.v. ancilla dei. Tertuliano, autor cristiano a caballo entre el s. II y el s. III d. C., designa con el término ancilla (De cult. femin., I, 4, 2; II, 1,1) a las servidoras de Dios. En general a una mujer fiel y sumisa a Dios por oposición a ancilla diaboli, modo en que llama a las paganas (De cult. femin., II, 11,2).

50 A. DE LA BLAISE: Dictionnaire Latin-Français des autors chretiens. Thurnhout, 1954. s. v. ministra.

51 Paul dE NOLA: Ep., 32, 15; 13, 13.

52 Como lo recogen las Constituciones Apostólicas. Aparecen citadas en la jerarquía en el s. If d. C. Vid. Carroll-Lequero: DACL. Paris, 1922. s.v. «diaconesse». 